\title{
Descending Projections from the Paraventricular Hypothalamic Nucleus to the Spinal Cord: Anterograde Tracing Study in the Rat
}

\author{
By \\ Jun-Qi ZHENG, Makoto SEKI, Tetsu HAYAKAWA, Hisao ITO and Katuya ZYO \\ Department of Anatomy, Hyogo College of Medicine, Mukogawa-cho, Nishinomiya, Hyogo 663, Japan
}

- Received for Publication, May 1, $1995-$

\begin{abstract}
Key Words: Intermediolateral cell column, Sacral parasympathetic nucleus, Lateral funiculus, Laminae I and X, PHA-L anterograde tracing study

Summary: We investigated the direct projections from the paraventricular hypothalamic nucleus (PVH) to the spinal cord. When Phaseolus vulgaris leucoagglutinin (PHA-L) was injected into the PVH, labeled descending fibers were observed running bilaterally through three pathways. The first pathway ran into the dorsal longitudinal fasciculus and projected to the central gray matter, Edinger-Westphal nucleus, pedunculopontine tegmental nucleus, nucleus of the locus ceruleus and parabrachial nucleus. The second and third pathways coursed through the medial forebrain bundle, ventral tegmental area, and ventral part of the medulla oblongata. At the medulla oblongata, the second pathway curved dorsolaterally and joined Rexed's lamina $\mathrm{V}$ of $\mathrm{Cl}$ after giving many projections to the nucleus ambiguus, nucleus of the solitary tract, dorsal motor nucleus of the vagus, and a few to the area postrema. The fibers descended through lamina $V$ until C5, and coursed through lamina I from C6 to the upper coccygeal segments. They gave collateral projections to lamina I from the cervical through the upper coccygeal segments. The third pathway coursed laterally and descended through the lateral funiculus after giving projections to the lateral reticular nucleus and the marginal layer of the spinal trigeminal nucleus. These fibers gave off many projections to the intermediolateral cell column of the thoracic cord and the sacral parasympathetic nucleus. Lamina $X$ received many projections from the fibers of the lateral funiculus at $\mathrm{C5}$ through the b-segment of sacral spinal cord.

These results indicate that the PVH may integrate directly with the medullary and spinal autonomic regulatory nuclei, including the vagus complex, sympathetic intermediolateral cell column, laminae $I$ and $X$, and sacral parasympathetic nucleus.
\end{abstract}

The paraventricular hypothalamic nucleus (PVH) is composed of several functionally distinct subnuclei that affect both endocrine and autonomic responses (Swanson, 1977; Buijs, 1978; Nilaver et al., 1978; Armstrong et al., 1980; Pittman et al., 1981). The magnocellular nucleus of the PVH sends relatively short axons to the endocrine system of the pituitary gland (Sherlock et al., 1975; Swanson and Kuypers, 1980; Wiegand and Price, 1980; Kiss et al., 1983). The parvocellular nucleus of the PVH sends axons not only to the median eminence but to various nuclei in the brainstem and spinal cord, such as the parabrachial nucleus, the nucleus of the locus ceruleus, the dorsal motor nucleus of the vagus, the nucleus of the solitary tract, the intermediolateral cell column and the sacral parasympathetic nucleus (Hancock, 1976; Saper et al., 1976; Swanson, 1977; Cedarbaum and Aghajanian, 1978; Ono et al., 1978; Hosoya and Matsushita, 1979; Swanson and Mckellar,
1979; Holstege, 1987).

These nuclei regulate autonomic charges occurring in the sympathetic and parasympathetic outflow system. The PVH receives general visceral and autonomic information from the bed nucleus of the stria terminalis, the septal nucleus, the preoptic nuclei, the lateral hypothalamic nucleus, the amygdala, the parabrachial nucleus, and the nucleus of the solitary tract (Silverman et al., 1981; Sawchenko and Swanson, 1983; Fulwiler and Saper, 1984; Cunningham and Sawchenko, 1988; Simerly and Swanson, 1988; Ter Horst et al., 1989; Cullinan et al., 1993). For these reasons, the PVH is considered one of the major forebrain autonomic structures.

Many retrograde tracing studies using horseradish peroxidase (HRP) or fluorescent dyes have revealed direct projections of the PVH to the cervical, thoracic and lumbar spinal cord (Ono et al., 1978; Hosoya

Correspondence to: Makoto SEKI, Department of Anatomy, Hyogo College of Medicine, Mukogawa-cho, Nishinomiya, Hyogo 663, Japan 
and Matsushita, 1979; Swanson and Kuypers, 1980; Tucker and Saper, 1985). These studies, however, checked only specific segments at different levels in the spinal cord. Luiten et al. (1985) showed, in an anterograde tracing study, that there were two descending pathways from the PVH in the brainstem. However, they did not adequately describe the descending fibers in the lower medulla and spinal cord. Swanson and Kuypers (1980) suggested that most of the PVH neurons projecting to the spinal cord were different from those projecting to the vagal nuclear complex.

The present study was attempted to investigate the route of the PVH descending fibers and the projection region in the medulla and in each segment of the spinal cord, using the anterograde tracing method of Phaseolus vulgaris leucoagglutinin (PHA-L).

\section{Materials and Methods}

Twenty two male Wistar rats were used in this study. All surgical procedures were carried out under sodium pentobarbital $(30 \mathrm{mg} / \mathrm{kg}$, i.p.) anesthesia.

For the anterograde tracing studies, restricted PHA-L injections were made into the PVH. A fine tip glass pipet (diameter $10-12 \mu \mathrm{m}$ ) was filled with $2.5 \%$ PHA-L (Seikagakukogyo, Tokyo) in $0.1 \mathrm{M}$ phosphate buffer at $\mathrm{pH}$ 7.4. The tracer was injected iontophoretically with a current of $10-20 \mu \mathrm{A}$, applied in an alternative mode (5 seconds on, 5 seconds off). After 10 to 14 days, the animals were perfused with $200 \mathrm{ml}$ saline, followed by $200 \mathrm{ml}$ of a solution containing $4 \%$ paraformaldehyde and $0.1 \%$ glutaraldehyde in $0.1 \mathrm{M}$ phosphate buffer, and then with $500 \mathrm{ml}$ of $4 \%$ paraformaldehyde. The brain and all segments of the spinal cord were removed, embedded in an agarose-gelatine mixture, and postfixed overnight with a $4 \%$ paraformaldehyde solution containing $20 \%$ sucrose. The brain and spinal cord were cut with a freezing microtome at $40 \mu \mathrm{m}$ and collected in a chilled $1 \%$ bovine serum solution in $0.1 \mathrm{M}$ Tris- $\mathrm{HCl}$ buffer containing $0.1 \%$ Triton-X100 and $0.9 \%$ sodium chloride at $\mathrm{pH} 7.4$.

Immunohistochemical procedures for PHA-L were carried out according to Gerfen and Sawchenko (1984). Every third section of the brainstem and all segments of the spinal cord was incubated for two days in rabbit anti-PHA-L antibodies (E-Y Labs, dilution 1:800). The primary antibodies were localized by incubation in biotinylated goat anti-rabbitIgG (Vector, dilution 1:2000), and in a commercially available $\mathrm{ABC}$ kit (Vector). The sections were rinsed in Tris-buffer solution containing Triton-X100 between each incubation. We employed the cobaltglucose oxidase method with $\mathrm{DAB}$ of Itoh et al.
(1979) to visualize the labeled fibers and terminallike boutons. After mounting on gelatin-coated slides, the sections were dehydrated, coverslipped and observed under bright or darkfield illuminations. Some sections containing labeled terminal-like boutons were dehydrated with ether-alcohol (1:1) and counterstained with thionin. We followed the terminology of Paxinos and Watson (1986) for the nuclei in the brainstem and the spinal cord, and the terminology of the Swanson and Kuypers (1980) for the subnucleus in the PVH.

\section{Results}

The injection of PHA-L was applied precisely to the PVH. The center of injection was distinguished as brown-black deposit. The center of the injection sites for 10 cases are shown in Figure 1. These included the dorsal, medial, lateral and posterior parvocellular divisions, and the posterior magnocellular division (Figs. 1, A, B). All cases of which injection sites included mainly the parvocellular part of PVH (Cases, P25, P26, P31, P33, P35), had descending labeled fibers and terminal-like boutons in the spinal cord (Table). The distribution pattern of the labeled fibers and terminal-like boutons was similar in these cases. But the cases of which injection sites included mainly the magnocellular part of PVH (Cases, P12, P19, P28, P29, $\mathrm{P} 32)$, had a few labeled descending fibers in the lower brainstem, and no labeled fibers in the spinal cord (Table). Because the parvocellular and magnocellular subnuclei are small, and have complex shapes, we could not show topographical descending projections for these subnuclei. The injection site of P35 was restricted to the medial parvocellular and the ventromedial edge of the posterior magnocellular parts (Figs. 1, A, C). Since the labeled descending fibers were many and conspicuous in case P35, we showed this as a typical case (Fig. 2). The descending fibers from the PVH initially divided into two main pathways, that is a dorsal and a ventral pathways. Many labeled fibers from the injection site ran along the contralateral side through the periventricular gray of the third ventricle, although the density of labeled fibers was greater on the ipsilateral side.

The fibers of the dorsal pathway descended along the periventricular region and entered the dorsal longitudinal fasciculus (Figs. 2, A-E). There were many bilateral fibers and terminal-like boutons in the Edinger-Westphal nucleus, the ventral part of the midbrain central gray matter, the pedunculopontine tegmental nucleus, the dorsal raphe nucleus, the parabrachial nucleus, and the nucleus of the locus ceruleus (Figs 2, C-E). The number of labeled 
Table Distribution of anterogradely labeled terminal-like boutons in the midbrain pons, medulla oblongata and spinal cord after PHA-L injection into the paraventricular hypothalamic nucleus.

\begin{tabular}{|c|c|c|c|c|c|c|c|c|c|c|}
\hline \multirow[b]{2}{*}{ Nuclei } & \multicolumn{5}{|c|}{ Parvocellular part } & \multicolumn{5}{|c|}{ Magnocellular part } \\
\hline & $\mathrm{P} 25$ & $\mathrm{P} 26$ & P31 & P33 & P35 & $\mathbf{P} 12$ & P19 & $\mathrm{P} 28$ & P29 & P32 \\
\hline Midbrain central gray matter & +++ & +++ & +++ & ++ & +++ & + & ++ & ++ & + & ++ \\
\hline Edinger-Westphal $\mathrm{n}$. & + & + & + & + & + & + & + & + & - & + \\
\hline Interfascicular $\mathrm{n}$. & + & + & + & + & ++ & + & + & + & + & + \\
\hline Rostral linear $\mathrm{n}$. & + & + & + & + & $+t$ & + & + & + & + & - \\
\hline Lateral lemniscus $\mathrm{n}$. & ++ & + & ++ & + & +++ & + & + & + & - & + \\
\hline Pedunculopontine tegmental $\mathrm{n}$. & ++ & ++ & + & + & +++ & + & + & + & + & + \\
\hline Median raphe $\mathrm{n}$. & + & ++ & ++ & + & ++ & + & + & + & + & + \\
\hline Dorsal raphe $\mathrm{n}$. & + & ++ & ++ & + & ++ & + & + & + & + & + \\
\hline Locus ceruleus $\mathrm{n}$. & ++ & ++ & ++ & ++ & ++ & + & + & + & + & + \\
\hline Parabrachial n. & ++ & ++ & ++ & ++ & ++ & + & + & + & + & + \\
\hline Raphe magmus $\mathrm{n}$. & + & + & ++ & + & ++ & + & + & + & + & + \\
\hline Raphe pallidus $\mathrm{n}$. & + & + & ++ & + & ++ & + & + & + & + & + \\
\hline Lateral reticular $n$. & ++ & + & ++ & + & ++ & - & - & - & - & - \\
\hline Ambiguous $\mathbf{n}$. & + & + & + & + & + & - & + & + & - & + \\
\hline Lateral paragigantocellular $\mathbf{n}$. & + & + & + & + & ++ & + & + & + & - & - \\
\hline Dorsal motor $n$. of vagus & +++ & ++ & +++ & ++ & +++ & + & + & + & + & + \\
\hline N. of the solitary tract & +++ & ++ & +++ & ++ & +++ & + & + & + & + & + \\
\hline Area postrema & + & + & + & - & + & - & - & - & - & - \\
\hline Spinal trigeminal $n$. & + & + & + & + & ++ & - & - & - & - & - \\
\hline Intermediolateral cell column & + & + & ++ & + & ++ & - & - & - & - & - \\
\hline Sacral parasympathetic $\mathrm{n}$. & + & + & ++ & + & ++ & - & - & - & - & - \\
\hline Lamina I of spinal cord & + & + & ++ & + & ++ & - & - & - & - & - \\
\hline Lamina $\mathrm{V}$ of spinal cord & + & + & + & + & ++ & - & - & - & - & - \\
\hline Lamina $X$ of spinal cord & + & + & +++ & + & +++ & - & - & - & - & - \\
\hline
\end{tabular}

$-;$ absence, $+;$ a few labeled terminal-like boutons, ++ ; many labeled terminal-like boutons, $+++;$ numerous labeled terminal-like boutons.

fibers and terminal-like boutons was slightly less on the contralateral side. There were a few collateral fibers and terminal-like boutons in the superior colliculus, the inferior colliculus, and the dorsal region of the midbrain central gray matter. A few labeled fibers scattered in the pontine reticular formation (Fig. 2, E). Only a few labeled fibers were seen in the surrounding areas of the dorsal tegmental nucleus. The labeled fibers in the dorsal longitudinal fasciculus ended in the dorsal tegmentum of the pons, and no labeled fibers were seen at the level of the facial genu (Figs. 2, E, F).

The fibers of the ventral pathway coursed through the medial forebrain bundle, ventral tegmental area, and along the interpeduncular nucleus. Some fibers branched off from this bundle, ran bilaterally along the dorsolateral aspect of the substantia nigra, and terminated in the nucleus of the lateral lemniscus (Fig. 2, C) and the ventral region of the pedunculopontine tegmental nucleus (Fig. 2, D). The major fibers of the ventral pathway run through the ventrolateral part of the pontine tegmentum and dorsal to the pyramidal tract (Figs. 2, D-H). Throughout their course, these fibers gave off terminal-like boutons in the nucleus linearis rostralis, the median raphe nucleus, the nucleus raphe magnus, and the nucleus raphe pallidus (Figs. 2, C-G).

At the medulla oblongata, the major fibers coursed through the ventromedial aspect of the superior olivary nucleus, and entered into the lateral paragigantocellular nucleus (Figs. 2, E-G). The fibers diverged bilaterally between the levels of the superior olivary nucleus and the lateral reticular nucleus (Figs. 2, E-H). Those branches ran along the dorsal aspect of the facial nucleus, and entered into the lateral reticular nucleus. A large number of labeled terminal-like boutons were found in the A5 noradrenaline cell group (Fig. 2, E) and the lateral region of the lateral reticular nucleus (Figs. 2, G, H: 3, A, B). A few, but distinctly labeled terminal-like boutons were seen at the ventral surface of the contralateral lateral paragigantocellular nucleus. At the level of the lateral reticular nucleus, the fibers of the ventral pathway again divided into lateral and dorsal bundles (Figs. 2, G, H).

After providing many terminal-like boutons to the lateral part of the lateral reticular nucleus (Fig. $3, \mathrm{~B})$, the fibers of the lateral bundle descended 
caudolaterally and passed through the dorsal part of the lateral funiculus of the spinal cord (Figs. 2, H, $\mathrm{C} 1$ ). Some fibers exited laterodorsally and gave terminal-like boutons in the ventral part of the marginal layer of the spinal trigeminal nucleus (Figs. $2, \mathrm{G}, \mathrm{H} ; 3, \mathrm{C})$. The dorsal bundle turned dorsomedially from the lateral reticular nucleus, traversed the nucleus ambiguus (Fig. 3, A) and the parvocellular reticular nucleus, and entered into the vagal nuclear complex (Fig. 3, E). Heavily labeled fibers and terminal-like boutons were observed bilaterally throughout the nucleus of the solitary tract and the dorsal motor nucleus of the vagus (Figs. 2, H; 3, D). There were a few labeled fibers and terminal-like boutons in the area postrema (Figs. 2, H; 3, D), as well as conspicuously labeled terminal-like boutons in the nucleus ambiguus and the $\mathrm{C} 1$ adrenaline cell group (Fig. 3, A). Some fibers branched off laterally and terminated in the dorsal region of the marginal layer of the spinal trigeminal nucleus (Figs. 2, G, H). After projecting to the vagal nuclear complex, fibers of the dorsal bundle turned laterally and entered Rexed's lamina V of the cervical spinal cord (Figs. 3, E, F).

In the spinal cord, fibers of the dorsal bundle descended mainly through lamina $V$ from the level of $\mathrm{C} 1$ to $\mathrm{C} 5$. The fibers in lamina $\mathrm{V}$ diverged dorsolaterally along the medial edge of lamina IV and entered lamina I (Figs. 2, C1, C5) from the level of $\mathrm{C} 1$ to C5. Downward from the level of C5, the majority of the fibers descended through lamina I until the rostral half of the coccygeal segment. Only a few fibers remained to run in lamina IV at the thoracic and lumbar segments (Fig. 5, B). The labeled fibers descending through lamina I did not intermingle with the fibers of the lateral funiculus (Fig. 5, A, B). Many fibers and terminal-like boutons were present bilaterally in lamina $I$ in all segments from the cervical to the rostral half of the coccygeal cord (Figs. 2, C1-Co1; 4, A; 5, A, B; 6, C), although they were decreased on the contralateral side. We could not follow the fibers at the level of the caudal half of the coccygeal segment.

Many labeled fibers were observed bilaterally from the cervical through the caudal coccygeal segment in the lateral funiculus (Figs. 2, $\mathrm{C} 1-\mathrm{Co} 2 ; 5$, A, B; 6, A, C). These fibers were dominant on the ipsilateral side. From the level of $\mathrm{C} 5$ to the $\mathrm{c}$-segment of the sacral spinal cord (Sc), the labeled fibers branched medially to enter the gray matter (Figs. 2, C5-Sb). These fibers ran through lamina VII from C5 to T12 (Figs. 2, C5-T8), or through lamina VI from $\mathrm{L} 1$ to $\mathrm{Sb}$, and entered lamina $\mathrm{X}$ (Figs. 2, L2-Sb). Distinctly labeled fibers and terminal-like boutons were present in lamina $\mathrm{X}$ through $\mathrm{C} 5$ to the caudal end of the sacral spinal segment, with a particular large number of terminal-like boutons present from T8 to Sa (Figs. 2, T8-Sa; 4, B; 6, A). There were no labeled fibers running into the lamina I from the lateral funiculus (Fig. 5, A, B; 6, C).

At all levels between $\mathrm{T} 3$ and $\mathrm{L} 4$, the fibers projecting to lamina $\mathrm{X}$ branched off bilaterally and terminated in the intermediolateral cell column (Figs. 2, T3-L2; 4, C). Medium-sized elongated neurons received conspicuous lebeled terminal-like boutons (Fig. 4, D). There were fewer terminal-like boutons on the contralateral side. The sacral parasympathetic nucleus received a large number of terminal-like boutons from fibers projecting to lamina $X$ at all levels from L5 to Sb on both sides (Figs. 2, L6-Sb; 6 , A). The contralateral sacral parasympathetic nucleus received many terminal-like boutons although there were more on the ipsilateral side (Figs. 2, L6-Sa). Many beaded labeled terminal-like boutons were in close apposition to the mediumsized neurons in the sacral parasympathetic nucleus (Fig. 6, B). Only a few labeled fibers were present in the anterior horn of $\mathrm{Sa}$, and labeled terminal-like boutons were found there (Fig. 2, Sa). Although few in number distinct labeled fibers were observed descending through the lateral funiculus of the coccygeal spinal cords (Fig. 6, C). However, we could not find labeled terminal-like boutons in this area.

\section{Discussion}

Our results revealed that the PVH descending projection was composed of three pathways. The first ran through the dorsal longitudinal fasciculus and terminated in the pedunculopontine tegmental nucleus, the parabrachial nucleus and the nucleus of the locus ceruleus. Both the second and the third pathways descended first through the medial forebrain bundle and the ventral tegmentum. They sent collateral fibers to the nucleus of the lateral lemniscus and the pedunculopontine tegmental nucleus. At the medulla oblongata, the second pathway curved dorsolaterally and descended through lamina $\mathrm{V}$ or lamina I through the cervical to the coccygeal spinal cord. The fibers gave a large number of terminal-like boutons at the dorsal motor nucleus of the vagus, the nucleus of the solitary tract, and lamina I at all spinal segments. The third pathway coursed through the ventral medulla, entered the dorsal part of the lateral funiculus and descended to the lower end of the coccygeal spinal cord. These fibers gave a large number of terminal-like boutons at the intermediolateral cell column, lamina $X$, and the sacral parasympathetic nucleus (Fig. 7).

Retrograde tracing studies reported that the PVH projected directly to the cervical, thoracic and lumbar 
spinal cord (Ono et al., 1978; Hosoya and Matsushita, 1979; Hosoya, 1980; Swanson and Kuypers, 1980; Schwanzel-Fukuda et al., 1984; Tucker and Saper, 1985; Wagner and Clemens, 1991). Tucker and Saper (1985) found that there was no topographic organization between segments of the thoracic spinal cord and neuronal cell groups in the PVH. We found that the PVH projected not only to the cervical, thoracic, and lumbar regions, but also to the sacral spinal cord. The spinal projection neurons are mainly present in the parvocellular division. The magnocellula division also sends a few descending fibers to the medullar oblongata. Our results agree with these studies. We found heavy projections bilaterally at the medullary nuclei relating to the autonomic functions, including the dorsal motor nucleus of the vagus, and the nucleus of the solitary tract, and a few to the area postrema and the nucleus ambiguus. These results confirm previous anterograde and reterograde tracing studies (Saper et al., 1976; Swanson and Kuypers, 1980; Hosoya and Matsushita, 1981; Hosoya, 1985; Holstege, 1987). There were also projections at the $\mathrm{A} 5$ noradrenaline and $\mathrm{C} 1$ adrerenaline cell groups. Both $\mathrm{A} 5$ and $\mathrm{C} 1$ groups project to the sympathetic preganglionic neurons of the thoracic spinal cord (Ross et al., 1981; Goodchild et al., 1984), and show sensitive vasopressor and respiratory activity (McAllen, 1986a, b; Otake $e t$ al., 1990; Morrison et al., 1991). Thus, the PVH may regulate sympathetic vasomotor and respiratory activity via these cell groups.

There have been several studies on descending projections from the PVH using the anterograde autoradiographic method (Saper et al., 1976; Conrad and Pfaff, 1976; Berk and Finkelstein, 1982; Holstege, 1987). Holstege (1987) observed that the medial hypothalamic area, including the PVH, sent fibers to the medulla and spinal cord. The hypothalamic descending fibers were divided into medial and lateral streams, which innervated the vagal nuclear complex and spinal cord. These autoradiographic tracing studies, however, were not able to adequately delineate the route of the descending fibers and projection areas, because the injection sites of tritiated leucine were generally too large and labeling of fibers with silver grains was not appropriate to determine the precise pathways.

Luiten et al. (1985) reported the routes of descending projections of the PVH using the PHA-L anterograde tracing method. They indicated two major descending bundles. One fiber tract ran through the mesencephalic periaqueductal grey matter, turned laterally and entered the pedunculopontine tegmental nucleus and the lateral lemniscus. Some fibers projected to the parabrachial nucleus and the nucleus of the locus ceruleus through the central grey matter.
The other fiber bundle coursed through the medial forebrain bundle and the ventral tegmentum. Some fibers turned laterodorsally to the lateral lemniscus and projected to the reticular formation. At the pontine level, these two fiber tracts joined each other at the lateral lemniscus and descended through the medulla oblongata. After projecting to the vagal nuclear complex, the fibers descended through the dorsolateral funiculus and gave collateral projections to the intermediolateral cell column and lamina $X$ through the cervical to the lumbar spinal cord.

Our results were different from those described by Luiten et al. (1985). First, the fibers running through the periaqueductal grey matter did not turn ventrally, nor did they join the lateral lemniscus. They generally terminated in the nucleus of the locus ceruleus and the parabrachial nucleus. Some fibers were present in the pontine reticular formation and projected to the pedunculopontine tegmental nucleus, but never traversed it. These discrepancies may have arisen when fibers from the periaqueductal grey matter were confused with those from the ventral tegmentum at the pedunculopontine tegmental nucleus. We believe that the main fiber tracts projecting to the spinal cord course through the medial forebrain bundle, the ventral tegmentum along the interpeduncular nucleus, and the ventrolateral part of the pontine tegmentum to the medulla oblongata.

A second discrepancy involves their description of all spinal projection fibers running through the dorsolateral funiculus. We found that the fibers after projecting to the vagal nuclear complex turned caudolaterally and entered lamina V (Figs 3, E, F), then gave terminal-like boutons to lamina $l$ at all levels of the spinal cord (Fig. 5, A, B). Some of the fibers projected to the spinal trigeminal nucleus. Even at the coccygeal spinal cord, there were labeled terminal-like boutons in lamina I (Fig. 6, C). There were no fibers coming from the lateral funiculus to lamina I. This is an important finding of our study. It was made possible by use of a long survival time of the PHA-L tracer. These results suggest that PVH neurons regulate nociceptive and somatosensory inputs to all parts of the body (Brown, 1981). Fibers running in the lateral reticular nucleus joined the dorsolateral funiculus, and gave terminal-like boutons to all regions of the intermediolateral cell column and sacral parasympathetic nucleus. This agrees with studies by Holstege (1987) and Schwanzel-Fukuda et al. (1984). In the cat there were some labeled neurons in Onuf's nucleus (Holstege, 1987). Although we observed only a few labeled fibers, there were no terminal-like boutons in the ventral horn of the sacral spinal cord. As Onuf's nucleus is not prominent in the rat, few fibers of the PVH project to this nucleus. 
The interesting aspect of this study is that lamina $\mathrm{X}$ received many terminal-like boutons from $\mathrm{C} 5$ to Sc. Neurons of lamina $X$ contain noxious specific cells as well as sympathetic preganglionic neurons (Nahin et al., 1983; Ugolini, 1992). Thus the PVH neurons in the dorsolateral funiculus may regulate sympathetic, parasympathetic as well as nociceptive inputs in this region (De Groat et al., 1981; Nahin et al., 1983; Ugolini, 1992). There were also labeled fibers in the lateral funiculus at the coccygeal spinal cord, but no labeled terminal-like boutons there. Swanson and Mckellar (1979) showed oxytocin containing fibers and terminal-like boutons in the coccygeal spinal cord and the filum terminale. Then these labeled fibers may terminate at the filum terminale.

The PVH neurons contain many kinds of neurotransmitters, such as oxytocin, vasopressin, somatostatin, dopamine, enkephalin, neurotensin, cholecystokinin, dynorphin, substance $\mathrm{P}$ and corticotropin releasing factor (see Swanson and Sawchenko, 1983 for a review). Double labeling studies of retrograde tracing and immunohistochemistry revealed that a few neurons of the PVH projecting to the parabrachial nucleus contain enkephalin (2\%), dynorphin $(1 \%)$, corticotropin releasing factor (CRF, less than $1 \%$ ), galanin (less than $1 \%$ ) and neurotensin (less than $1 \%$ ). A few oxytocin containing neurons project to the parabrachial nucleus (Moga et al., 1990). Neurons projecting to the thoracic spinal cord contain many vasopressin (35\%), oxytocin $(25 \%)$ and enkephalin $(10 \%)$ (Cechetto and Saper, 1988). About one-third of the neurons projecting to the vagal nuclear complex or the thoracic spinal cord contain vasopressin and corticotropin releasing factor (Sawchenko, 1987).

Immunohistochemical studies have described the descending pathway of neurophysin or oxytocin containing fibers (Swanson and Mckellar 1979). These immunopositive fibers distributed to the dorsomedial medulla including the vagal nuclear complex, the lateral reticular nucleus, the dorsolateral funiculus at the cervical to the coccygeal spinal cord, the intermediolateral cell column, the sacral parasympathetic nucleus, lamina $X$ and lamina $I$. Thus, it may be concluded that the PVH neurons project to three different regions. Neurons with oxytocin and vasopressin project to the medullary parasympathetic and spinal nociceptive neurons including the vagal nuclear complex and lamina I. Neurons with oxytocin and vasopressin project to the sympathetic and parasympathetic preganglionic spinal neurons including the intermediolateral cell column, the sacral parasympathetic nucleus and lamina $\mathrm{X}$. Neurons with little oxytocin and vasopressin project to the midbrain limbic or extra pyramidal motor neurons in- cluding the pedunculopontine tegmental nucleus, the nucleus of the locus ceruleus and the parabrachial nucleus. Although the present study showed that the PVH descending fibers coursed three different pathways, it is unclear whether a single neuron of the PVH sent collateral fibers to these three regions. Using retrograde double labeling of two kinds of fluorescent dyes, Swanson and Kuypers (1980) reported that only $10 \%$ of the $\mathrm{PVH}$ neurons project to both the vagal nuclear complex and the spinal cord. Thus, the PVH may contain three kinds of neurons projecting to these three different regions.

\section{Acknowledgements}

We thank Drs. Y. Kuroda and S. Doi for helpful comments and criticism of this manuscript. We are also grateful to Ms. M. Hatta and Mr. N. Okamura for secretarial help.

\section{References}

1) Armstrong WE, Warach S, Hatton GI and McNeill TH Subnuclei in the rat hypothalamic paraventricular nucleus: a cytoarchitectural, horseradish peroxidase and immunocytochemical analysis. Neurosci. 1980; 5:1931-1958.

2) Berk ML and Finkelstein JA. Efferent connections of the lateral hypothalamic area of the rat: An autoradiographic investigation. Brain Res. Bull. 1982; 8:511-526.

3) Brown AG. Organization in the spinal cord. Berlin: 1981; Springer-Verlag.

4) Buijs RM. Intra-and extrahypothalamic vasopressin and oxytocin pathway in the rat. Cell. Tiss. Res. 1978; 192 423-435.

5) Cechetto DF and Saper CB. Neurochemical organization of the hypothalamic projection to the spinal cord in the rat J. Comp. Neurol. 1988; 272:579-604.

6) Cedarbaum JM and Aghajanian GK. Afferent projections to the rat locus coeruleus as determined by a retrograde tracing technique. J. Comp. Neurol. 1978; 178:1-16.

7) Conrad LCA and Pfaff DW. Efferents from medial basal forebrain and hypothalamus in the rat. II An autoradiographic study of the anterior hypothalamus. J. Comp. Neurol. 1976; 169::221-262.

8) Cullinan WE, Herman JP and Watson SJ. Ventral subicular interaction with the hypothalamic paraventricular nucleus: Evidence for a relay in the bed nucleus of the stria terminalis. J. Comp. Neurol. 1993; 332:1-20.

9) Cunningham ET Jr and Sawchenko PE. Anatomical specificity of noradrenergic inputs to the paraventricular and supraoptic nuclei of the rat hypothalamus. J. Comp. Neurol. 1988; 274:60-76.

10) De Groat WC, Nadelhaft I, Milne RJ, Booth AM, Morgan $\mathrm{C}$ and Thor $\mathrm{K}$. Organization of the sacral parasympathetic reflex pathway to the urinary bladder and large intestine J. Autonom. Nervous Syst. 1981; 3:135-160.

11) Fulwiler CE and Saper CB. Subnuclear organization of the efferent connections of the parabrachial nucleus in the rat. Brain Res. Rev. 1984; 7:229-259.

12) Gerfen CR and Sawchencko PE. An anterograde neuroanatomical tracing method that shows the detailed 
morphology of neurons, their axons and terminals: immunohistochemical localization of an axonally transported plant lectin, Phaseolus vulgaris leucoagglutinin (PHA-L). Brain Res. 1984; 290:219-238.

13) Goodchild AK, Moon EA, Dampney DAL and Howe PRC. Evidence that adrenaline neurons in the rostral ventrolateral medulla have a vasopressor function. Neurosci. Lett. 1984; 45:267-272.

14) Hancock MB. Cells of origin of hypothalamo-spinal projections in the rat. Neurosci. Lett. 1976; 3:179-184.

15) Holstege G. Some anatomical observations on the projections from the hypothalamus to brainstem and spinal cord: An HRP and autoradiographic tracing study in the cat. J. Comp. Neurol. 1987; 260:98-126.

16) Hosoya $Y$. The distribution of spinal projection neurons in the hypothalamus of the rat, studied with the HRP method. Exp. Brain Res. 1980; 40:79-87.

17) Hosoya Y. Hypothaiamic projections to the ventral medulla oblongata in the rat, with special reference to the nucleus raphe pallidus: a study using autoradiographic and HRP techniques. Brain Res. 1985; 344:338-350.

18) Hosoya $Y$ and Matsushita $M$. Identification and distribution of the spinal and hypophyseal projection neurons in the paraventricular nucleus of the rat. A light and electron microscopic study with the horseradish peroxidase method. Exp. Brain Res. 1979; 35:315-331.

19) Hosoya $Y$ and Matsushita M. A direct projection from the hypothalamus to the area postrema in the rat, as demonstrated by the HRP and autoradiographic methods. Brain Res. 1981; 214:144-149.

20) Itoh $K$, Konishi A, Nomura S, Mizuno N, Nakamura $Y$ and Sugimoto T. Application of coupled oxidation reaction to electron microscopic demonstration of horseradish peroxidase: Cobalt-glucose oxidase methed. Brain Res. 1979; 175:341-346.

21) Kiss JZ, Palkovits M, Zaborszky L, Tribollet E, Szabo D and Makara GB. Quantitative histological studies on the hypothalamic paraventricular nucleus in rats. II Number of local and certain afferent nerve terminais. Brain Res. 1983; 265:11-20.

22) Luiten PGM, ter Horst GJ, Karst $\mathbf{H}$ and Steffens AB. The course of paraventricular hypothalamic efferents to autonomic structures in medulla and spinal cord. Brain Res. 1985; 329:374-378.

23) McAllen RM. Location of neurones with cardiovascular and respiratory function, at the ventral surface of the cat's medulla. Neurosci. 1986a; 18:43-49.

24) McAllen RM. Action and specificity of ventral medullary vasopressor neurones in the cat. Neurosci. 1986b; 18: 51-59.

25) Moga MM, Saper CB and Gray TS. Neuropeptide organization of the hypothalamic projection to the parabrachial nucleus in the rat. J. Comp. Neurol. 1990; 295:662-682.

26) Morrison SF, Callaway J, Milner TA and Reis DJ. Rostral ventrolateral medulla: a source of the glutamatergic innervation of the sympathetic intermediolateral nucleus. Brain Res. 1991; 562:126-135.

27) Nahin RL, Madsen A and Giesler GJ Jr. Anatomical and physiological studies of the gray matter surrounding the spinal cord central canal. J. Comp. Neurol. 1983; 220: $321-335$.

28) Nilaver G, Wilkins J, Michaels J, Hoffman DL, Silverman AJ and Zimmerman EA. Oxytocin-neurophysin pathways to the lower brainstem and spinal cord of the rat. Soc. Neurosci. Abst. 1978; 4:351.

29) Ono $T$, Nishino $H$, Sasaka $K$, Muramoto $K$, Yano $I$ and Simpson A. Paraventricular nucleus connections to spinal cord and pituitary. Neurosci. Lett. 1978; 10:141 - 146.

30) Otake K, Sasaki H, Ezure K and Manabe M. Medullary projection of nonaugmenting inspiratory neurons of the ventrolateral medulla in the cat. J. Comp. Neurol. 1990; 302:485-499.

31) Paxinos $G$ and Watson $C$. The Rat Brain in Stereotaxic Coordinates. 2nd edn. 1986; Sydney: Academic Press.

32) Pittman QJ, Blume HW and Renaud LP. Connections of the hypothalamic paraventricular nucleus with the neurohypophysis, median eminence, amygdala, lateral septum and midbrain periaqueductal gray: an electrophysiological study in the rat. Brain Res. 1981: 215:15-28.

33) Ross CA, Armstrong DM, Ruggiero DA, Pickel VM, Joh TH and Reis DJ. Adrenaline neurons in the rostral ventrolateral medulla innervate thoracic spinal cord: A combined immunocytochemical and retrograde transport demonstration. Neurosci. Lett. 1981; 25:257-262.

34) Saper CB, Loewy AD, Swanson LW and Cowan WM. Direct hypothalamo-autonomic connections. Brain Res. 1976; 117:305-312.

35) Sawchenko PE. Evidence for differential regulation of corticotropin-releasing factor and vasopressin immunoreactivities in parvocellular neurosecretory and autonomicrelated projections of the paraventricular nucleus. Brain Res. 1987; 437:253-263.

36) Sawchenko PE and Swanson LW. The organization of forebrain afferents to the paraventricular and supraoptic nuclei of the rat. J. Comp. Neurol. 1983; 218:121-144.

37) Schwanzel-Fukuda M, Morrell JI and Pfaff DW. Localization of forebrain neurons which project directly to the medulla and spinal cord of the rat by retrograde tracing with wheat germ agglutinin. J. Comp. Neurol. 1984; 226:1-20.

38) Sherlock DA, Field PM and Raisman G. Retrograde transport of horseradish peroxidase in the magnoccllular neurosecretory system of the rat. Brain Res. 1975; 88: 403-414.

39) Silverman AJ, Hoffman DL and Zimmerman EA. The descending afferent connections of the paraventricular nucleus of the hypothalamus (PVN). Brain Res. Bull. $1981 ; 6: 47-61$.

40) Simerly RB and Swanson LW. Projections of the medial preoptic nucleus: A Phaseolus vulgaris Leucoagglutinin anterograde tract-tracing study in the rat. J. Comp. Neurol. 1988: 270:209-242.

41) Swanson LW. Immunohistochemical evidence for a neurophysin-containing autonomic pathway arising in the paraventricular nucleus of the hypothalamus. Brain Res. 1977; 128: 346-353.

42) Swanson LW and Mckellar S. The distribution of oxytocinand ncurophysin-stained fibers in the spinal cord of the rat and monkey. J. Comp. Neurol. 1979; 188:87-106.

43) Swanson LW and Kuypers HGJM. The paraventricular nucleus of the hypothalamus: Cytoarchitectonic subdivisions and organization of projections to the pituitary. dorsal vagal complex, and spinal cord as demonstrated by retrograde fluorescence double-labcling methods. J. Comp. Ncurol. $1980 ; 194: 555-570$

44) Swanson LW and Sawchenko PE. Hypothalamic integration: Organization of the paraventricular and supraoptic nuclei. Ann. Rev. Neurosci. 1983; 6:269-324.

45) Ter Horst de Boer GJP, Luiten PGM and van Willigen JD. Ascending projections from the solitary tract nucleus to the hypothalamus. A phascolus vulgaris lectin tracing study in the rat. Neurosci. 1989; 31:785-797.

46) Tucker DC and Saper CB. Specificity of spinal projections from hypothalamic and brainstem arcas which innervate sympathetic preganglionic neurons. Brain Res. 1985; 360: 
159-164.

47) Ugolini G. Transneuronal transfer of herpes simplex virus type 1 (HSV1) from mixed limb nerves to the CNS. 1 . Sequence of transfer from sensory, motor, and sympathetic nerve fibers to the spinal cord. J. Comp. Neurol. 1992; 326: 527-548.

48) Wagner CK and Clemens LG. Projections of the paraventricular nucleus of the hypothalamus to the sexually dimorphic lumbosacral region of the spinal cord. Brain Res. 1991; 539:254-262.

49) Wiegand SJ and Price JL. Cells of origin of the afferent fibers to the median eminence in the rat. J. Comp. Neurol. 1980: 192:1-19.

\section{Abbreviations for Figures}

\begin{tabular}{ll} 
5SPN & spinal trigeminal nucleus \\
7 genu of the facial nerve \\
$7 \mathrm{~N}$ & facial nucleus \\
A5 & A5 noradrenergic cell group \\
AHP & anterior hypothalamic area \\
Amb & ambiguus nucleus \\
AP & area postrema \\
Ar & arcuate hypothalamic nucleus \\
C1, C5, C8 & 1st, 5th and 8th segments of cervical spinal \\
CC & cord \\
CGM & central canal \\
Co1 & midbrain central gray matter \\
Co2 & upper segment of coccygeal spinal cord \\
DMX & lower segment of coccygeal spinal cord \\
dp & dorsal motor nucleus of the vagus \\
DRN & dorsal parvocellular part of PVH \\
DTg & dorsal raphe nucleus \\
E-W & dorsal tegmental nucleus \\
f & Edinger-Westphal nucleus \\
IC & fornix \\
ic & inferior colliculus \\
\hline
\end{tabular}

IF

IML

IP

L2, L6

LC

Lfu

LH

LL

Ip

LPGi

LRN

$\mathrm{ml}$

mlf

mp

MRN

$\mathrm{mt}$

PBN

pm

PPT

pv

PV

PVH

$\mathrm{Re}$

$\mathrm{RLi}$

$\mathrm{RMg}$

$\mathrm{RPa}$

I, IV, V, VI, VII, X

$\mathrm{Sa}, \mathrm{Sb}, \mathrm{Sc}$

scp

SNR

Sol

SO

SPN

T3, T8

V3

VMH interfascicular nucleus

intermediolateral cell column

interpeduncular nucleus

2nd and 6th segments of lumbar spinal cord

nucleus of the locus ceruleus

lateral funiculus

lateral hypothalamic nucleus

nucleus of the lateral lemniscus

lateral parvocellular region of $\mathrm{PVH}$

lateral paragigantocellular nucleus

lateral reticular nucleus

medial lemniscus

medial longitudinal fasciculus

medial parvocellular part of $\mathrm{PVH}$

median raphe nucleus

mamillothalamic tract

parabrachial nucleus

posterior magnocellular part of $\mathrm{PVH}$

pedunculopontine tegmental nucleus

periventricular part of PVH

paraventricular thalamic nucleus

paraventricular hypothalamic nucleus

reuniens thalamic nucleus

rostral linear nucleus of the raphe

raphe magnus nucleus

raphe pallidus nucleus

Rexed's laminae I, IV, V, VI, VII and X of spinal cord

segments $\mathrm{a}, \mathrm{b}$ and $\mathrm{c}$ of sacral spiral cord superior cerebellar peduncle

substantia nigra, pars reticularis

nucleus of the solitary tract

superior olivary nucleus

sacral parasympathetic nucleus

3rd and 8th segments of thoracic spinal cord

3rd ventriclc

ventromedial hypothalamic nucleus 

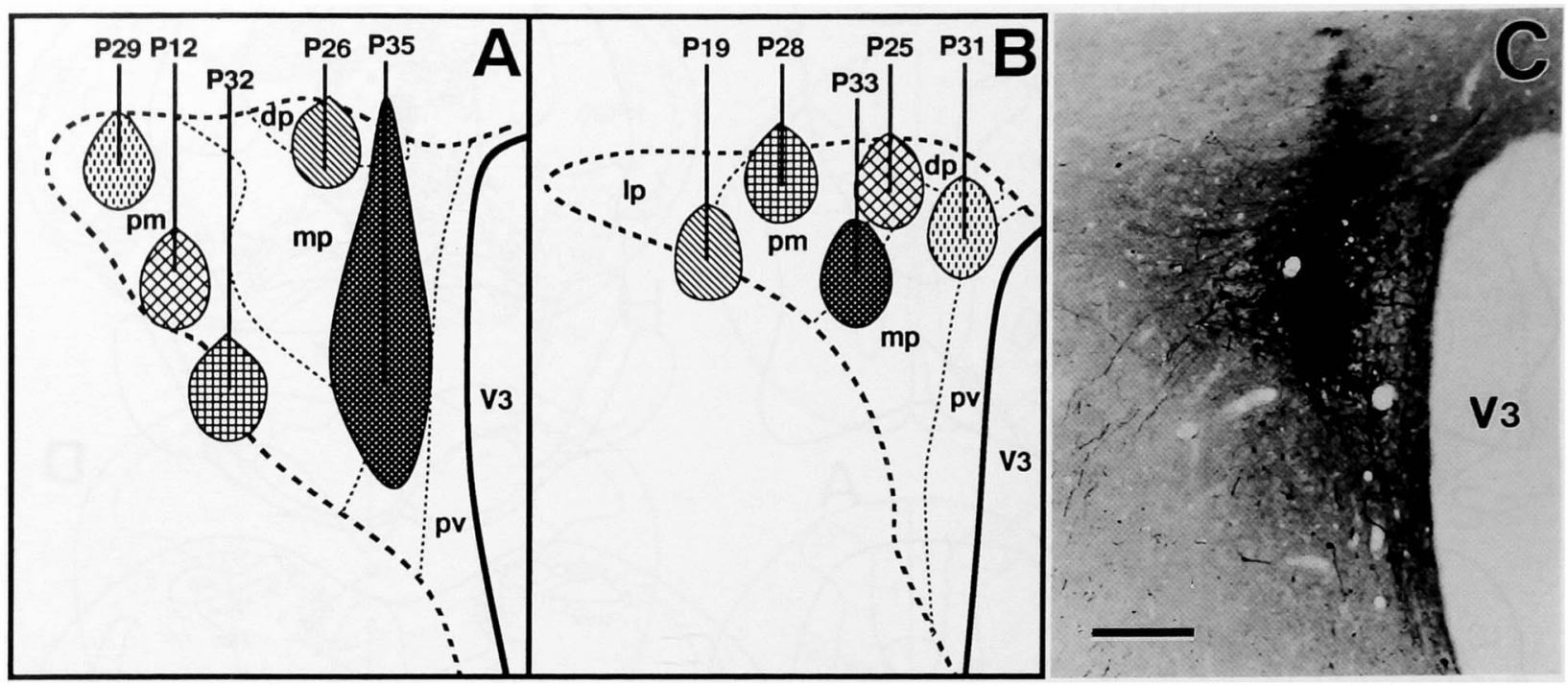

\section{Explanation of Figures}

\section{Plate I}

Figs. 1A and B. Schematic representation of PHA-L injection sites (shaded area) in the middle (A) and caudal (B) region of the paraventricular hypothalamic nucleus. C. Light photomicrograph of the PHA-L injection site in the middle part of the paraventricular hypothalamic nucleus in Case P35. Bar is $100 \mu \mathrm{m}$ in C. 
Plate II
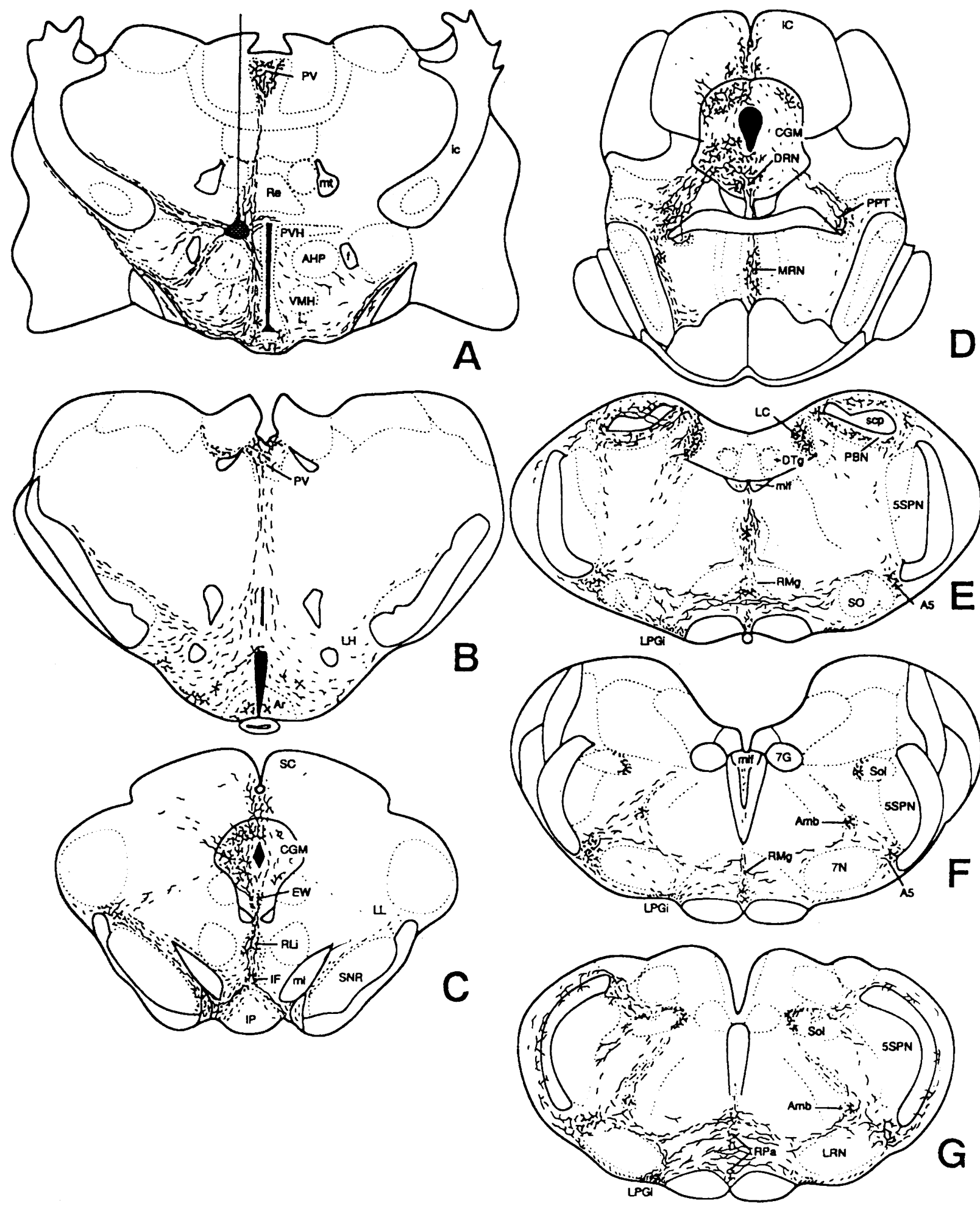

Plate II

Figs. 2 A-Co2. Schematic representation of the distribution of PHA-L labeled fibers (dashed lines) and terminal-like boutons (dots) in the brainstem and spinal cord after injection into the middle part of the paraventricular hypothalamic nucleus (Case P35). 


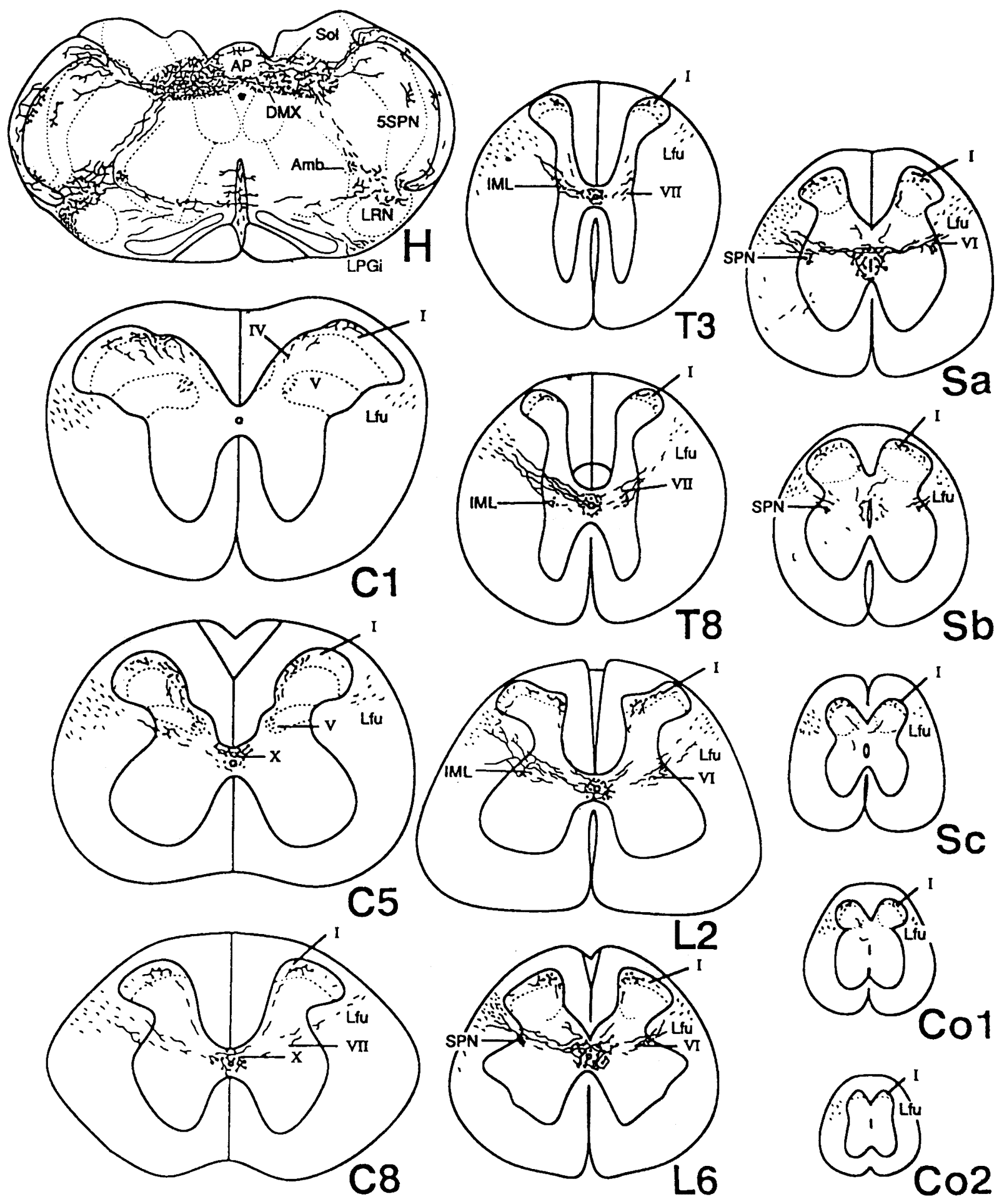




\section{Plate III}

Fig. 3. Photomicrograph of PHA-L labeled fibers and terminal-like boutons. A. Labeled terminal-like boutons in the ambiguus nucleus. Note PHA-L labeled fibers curving dorsomedially (arrows) to the vagal nuclear complex. B. PHA-L labeled fibers and terminal-like boutons in the lateral reticular nucleus. C. PHA-L labeled fibers and terminal-like boutons in the marginal area of the spinal trigeminal nucleus. D. Dense PHA-L labeled fibers and terminal-like boutons in the bilateral nucleus of the solitary tract and the dorsal motor nucleus of the vagus. Note a few labeled fibers in the area postrema. E. The PHA-L labeled fibers enter the ventrolateral region (arrow) and exit from the lateral part of the vagal nuclear complex (double arrow). F. PHA-L labeled fibers in lamina V (V) of the uppermost segment of the cervical cord. Double arrow indicates fibers running from the vagal nuclear complex (arrow) to lamina V. Bar is $100 \mu \mathrm{m}$ in $\mathrm{A}, \mathrm{B}, \mathrm{C}, \mathrm{E}$ and $\mathrm{F}, 300 \mu \mathrm{m}$ in D. 

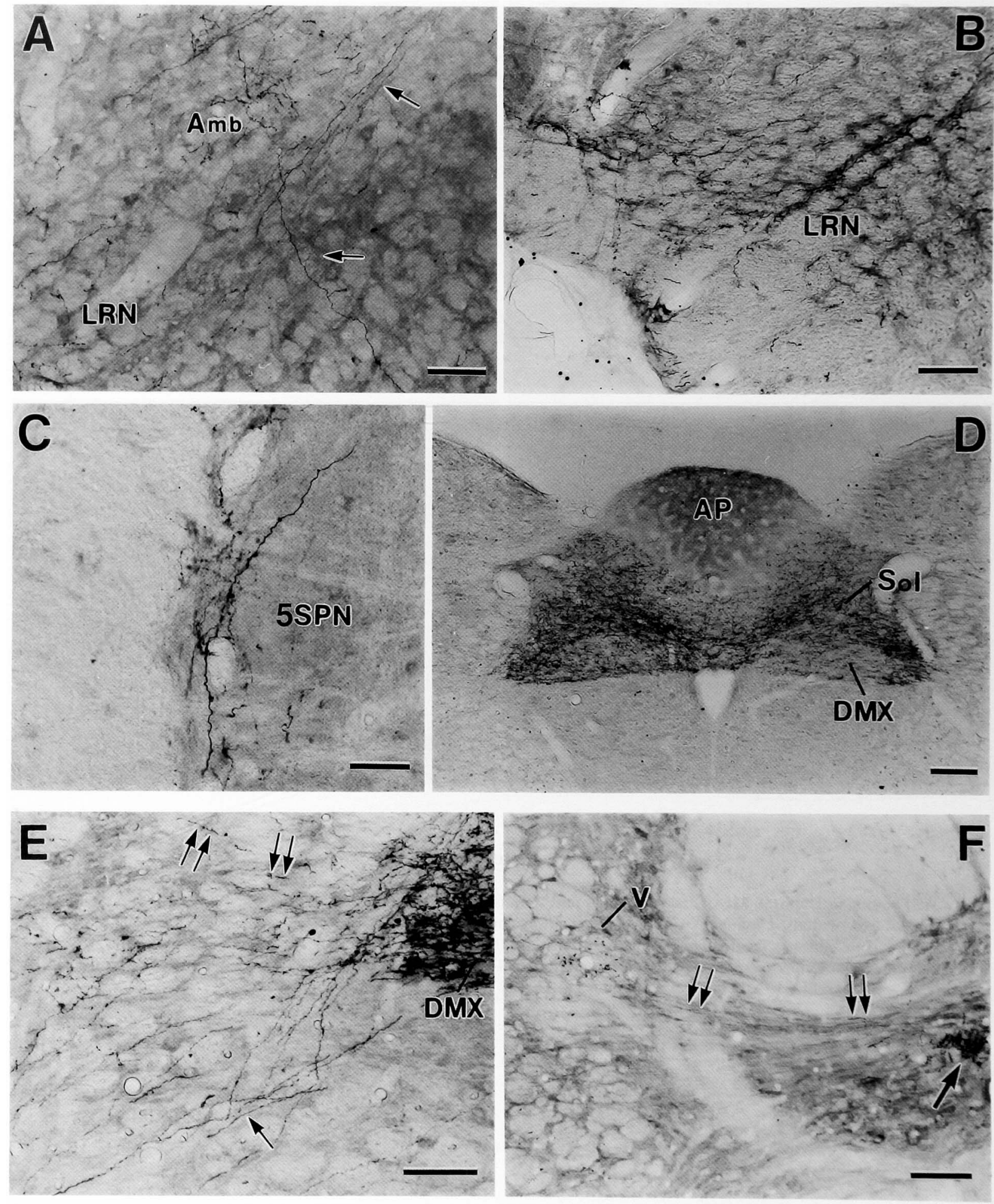
Plate IV
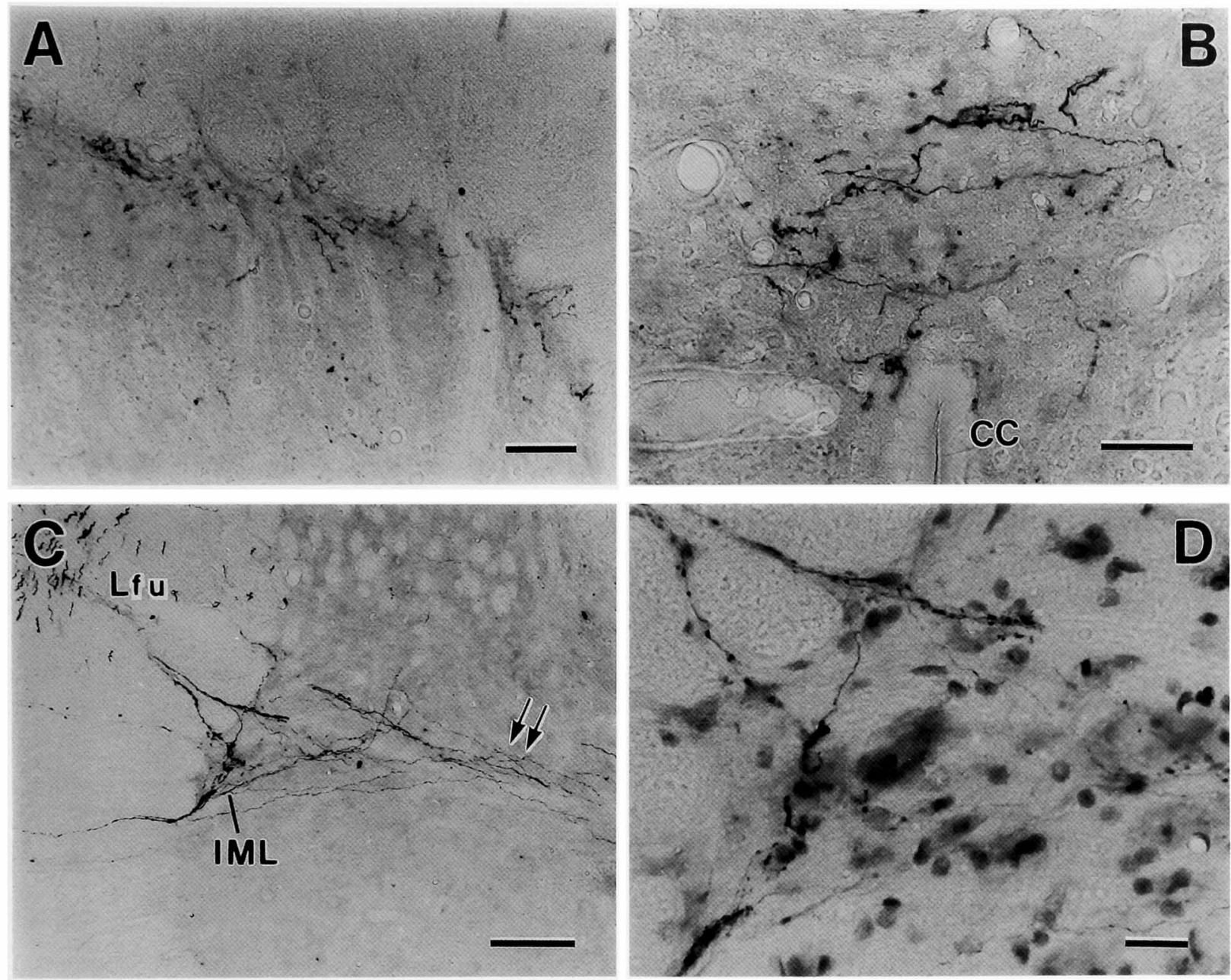

\section{Plate IV}

Fig. 4 A. Photomicrograph of PHA-L labeled fibers and terminal-like boutons in lamina I of C5. B. PHA-L labeled fibers and terminal-like boutons in lamina X of L2. C. Low power photomicrograph of the intermediolateral cell column (IML) of T8. Double arrow indicates labeled fibers running through the IML to lamina $\mathrm{X}$ after giving terminal-like boutons in the IML. D. High power photomicrograph of labeled fibers and terminal-like boutons in the IML of T8. Counterstained with thionin. Bar is $50 \mu \mathrm{m}$ in $\mathrm{A}$ and $\mathrm{B}, 100 \mu \mathrm{m}$ in $\mathrm{C}$, and $20 \mu \mathrm{m}$ in $\mathrm{D}$. 

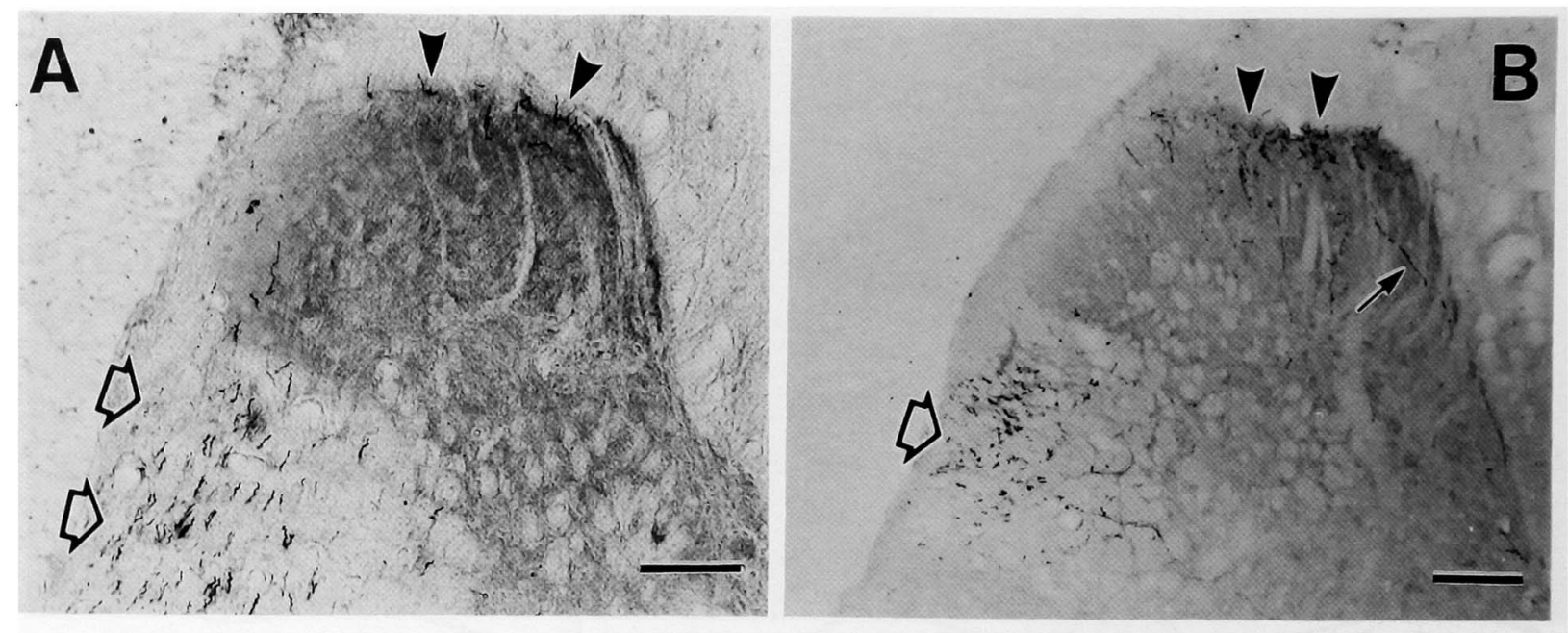

\section{Plate V}

Fig. 5A and B. Photomicrographs of two bundles of PHA-L labeled fibers running through lamina I (arrowheads) and the dorsal part of lateral funiculus (open arrows) at the level of T12 (A) and L4 (B) of spinal cord. Arrow indicates labeled fibers running from lamina V to lamina I. Bar is $100 \mu \mathrm{m}$ in $\mathrm{A}$ and $300 \mu \mathrm{m}$ in $\mathrm{B}$. 
Plate VI
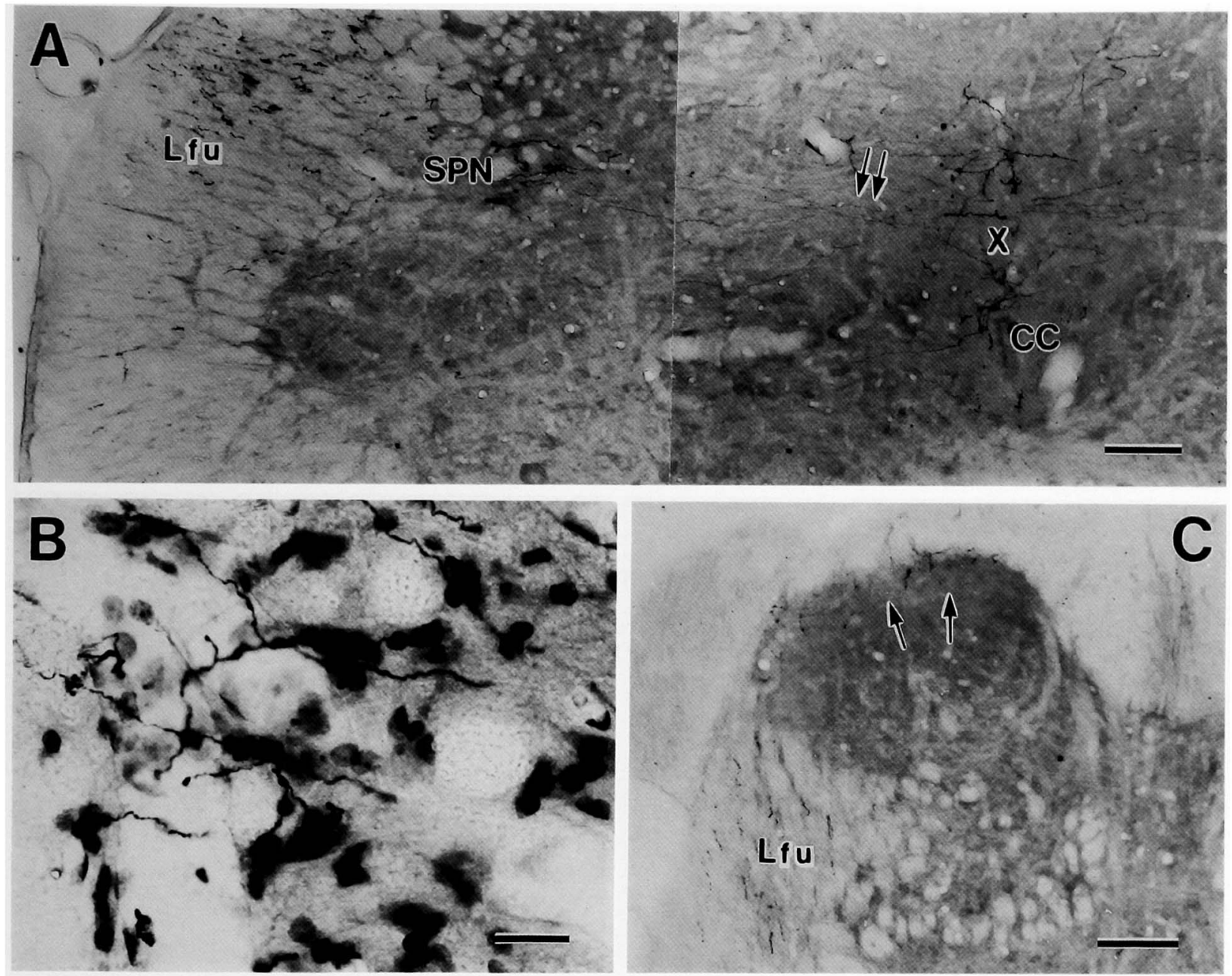

\section{Plate VI}

Fig. 6 A. Low power photomicrograph of PHA-L labeled fibers and terminal-like boutons in the a-segment of the sacral cord. Double arrow indicates labeled fibers running from the dorsal part of the lateral funiculus (Lfu) to lamina $X(X)$ after giving terminal-like boutons in the sacral parasympathetic nucleus (SPN). B. High power photomicrograph of PHA-L labeled fibers and terminallike boutons in the sacral parasympathetic nucleus of the a-segment of the sacral cord. Counterstained with thionin. C. Photomicrograph showing PHA-L labeled fibers in the lateral funiculus (Lfu) and terminal-like boutons in lamina I (arrows) of the coccygeal spinal cord. Bar is $100 \mu \mathrm{m}$ in A and C, $20 \mu \mathrm{m}$ in B. 


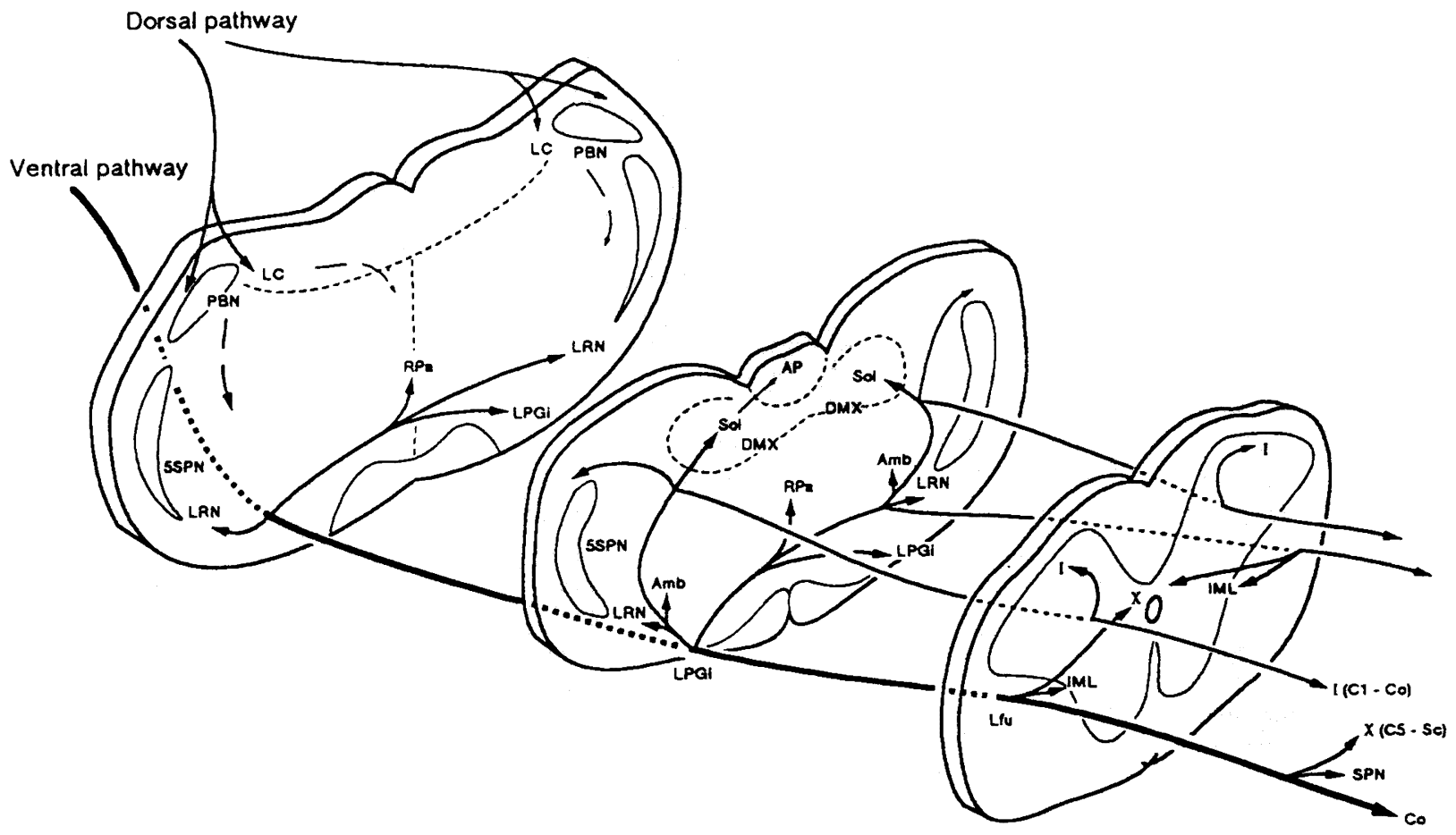

Plate VII

Fig. 7 Schematic representation of the main descending pathways from the paraventricular hypothalamic nucleus to the spinal cord based on our investigation. Note the descending projections to the spinal cord divided into two pathways at the level of the lateral reticular nucleus. 